\title{
Thoracoscopic Repair of Esophageal Atresia Through the Right Chest in Neonates with Right-Sided Aortic Arch
}

\author{
Kenneth K.Y. Wong, PhD, FRCSEd, FHKAM, and Paul K.H. Tam, ChM, FRCS, FRCPCH
}

\begin{abstract}
The management of neonates with esophageal atresia (EA) associated with right-sided aortic arch (RAA) is often difficult technically. Many surgeons would advise for repair through left-chest access. In this article, we report our experience of repairing 2 patients with EA associated with RAA thoracoscopically. We conclude that thoracoscopic repair is ideal for all patients with EA.
\end{abstract}

\section{Introduction}

$\mathbf{T}$ HE CARE OF NEONATES with esophageal atresia and tracheoesophageal fistula (EA/TEF) has seen a dramatic improvement over the past two decades, resulting in a significant improvement in the outcomes. For most infants, an immediate primary repair through thoracotomy is the usual method of treatment. However, a staged approach may be required for a few patients with hemodynamic instability. ${ }^{1}$ Traditionally, the repair of EA with TEF is ligation of fistula and primary anastomosis of both ends via open right thoracotomy. ${ }^{2}$ In a small proportion of infants, anatomic variation leads to the existence of right-sided aortic arch (RAA), which may obscure and obstruct the upper pouch of the esophagus., ${ }^{3,4}$ Thus, an unrecognized RAA found at thoracotomy may complicate the repair of EA and TEF. Many surgeons, therefore, advocate the routine use of echocardiography for patients with EA preoperatively, so as to determine the laterality of the aortic arch. ${ }^{5,6}$ In the discovery of right-sided arch, many surgeons would opt for access through a left-sided thoracotomy for esophageal repair. ${ }^{7,8}$ However, preoperative echocardiography has been shown to be unreliable and may fail to show RAA associated with EA/TEF. ${ }^{5,6}$

The recent introduction of the thoracoscopic approach for the repair of EA has dramatically improved both intraoperative stability and, also, postoperative outcomes. ${ }^{9}$ With thoracoscopy, the operative view is magnified significantly to help with dissection and anastomosis. However, no report exists on the thoracoscopic approach in the event of RAA. In this article, we report our series of using thoracoscopic repair for 2 patients with EA and RAA. We showed that the existence of RAA did not interfere with the right-sided approach. We, therefore, recommend the use of thoracoscopic repair for all patients with EA.

\section{Case 1}

A newborn baby boy was transferred to us from another hospital with a clinical diagnosis of EA with distal TEF. He was born at 39 weeks with a birth weight of $2.8 \mathrm{~kg}$. Antenatally, polyhydramnios was noted and a nasogastric tube could not be passed after birth. X-ray confirmed the diagnosis, with the tip of the nasogastric tube being at the T3/4 level. Routine echocardiography showed the presence of RAA. No other associated anomaly was noted. Since we were familiar with the thoracoscopic repair of EA, it was decided that we would approach this patient also from the right side. At the operation, the RAA was confirmed. The upper pouch of the esophagus was mobilized and brought anterior to the aorta and then anastomosed to the lower pouch after the ligation and division of the fistula. The baby made an excellent recovery and was discharged on day 18 after the operation.

\section{Case 2}

The second baby was also a boy with an antenatal history of polyhydramnios. He was born at 38 weeks by emergency caesarian section because of fetal distress. A copious amount of saliva was noted after birth, and EA was suspected. A nasogastric tube was attempted but could not be passed. A chest X-ray performed showed the tip of the nasogastric tube to be at the T4 level, with the presence of intestinal gas. Thus, EA with distal TEF was diagnosed. Echocardiography showed a 4-mm ventricular septal defect but no apparent RAA. Thirteen pairs of ribs were noted on X-ray. The ultrasonogram of the urinary system was normal.

Thoracoscopic repair of the EA was carried out the following day through the right side. At the operation, RAA, which had not been diagnosed preoperatively, was noted (Fig. 1). The repair was performed in the usual manner with

Department of Surgery, LKS Faculty of Medicine, The University of Hong Kong, Hong Kong, China. 


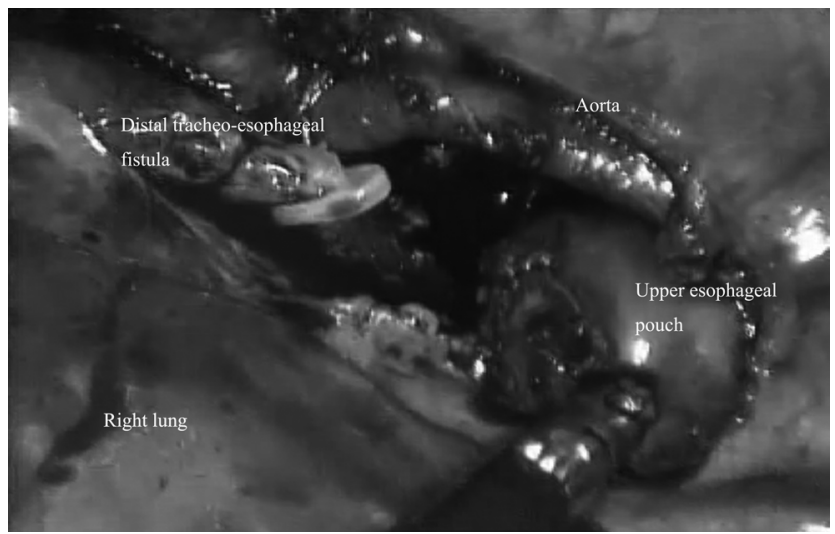

FIG. 1. A photo taken intraoperatively during thoracoscopic repair of esophageal atresia showing the presence of right-sided aortic arch.

no difficulty. The patient resumed oral feeding on day 5 and was discharged 2 weeks after the operation.

\section{Discussion}

EA and TEF may accompany various cardiac anomalies, including tetralogy of Fallot, transposition of great vessels, atrioventricular defects, and RAA. ${ }^{10}$ The incidence of RAA in combination with EA has been suggested to be found in between 5 and $13 \%$ of patients., ${ }^{3,4}$ For traditional open repair of EA, the presence of RAA has been reported by many researchers to increase the technical difficulty of the operation, due to the aortic arch being in front of the esophagus, and that a conversion to a left-sided thoracotomy may be needed. Although the presence of RAA is not a contraindication for the right-sided thoracic approach, many surgeons, including us in the past, would opt to gain access through the left thorax, if the diagnosis of RAA is known preoperatively. ${ }^{7,8}$ Indeed, in the series of 104 patients described by Holcomb et al., 6 patients who were found to have RAA, either pre- or postoperatively, all received their operative repair through the left chest..$^{9}$ As a result, echocardiography has been used to as a noninvasive technique to screen for its presence preoperatively. Despite this, the sensitivity of echocardiography is low, and, as illustrated in patient 2 in our series, the RAA was not diagnosed until during the operation. For patient 1, although we knew of the presence of RAA, we believed that, with a magnified view under thoracoscopic guidance, a rightsided approach was possible. This, indeed, was proven to be the case. Both patients underwent repair with no significant difficulty.

\section{Conclusions}

In conclusion, we show that thoracoscopic repair of EA is an advance in surgical technique, and access through the right chest is still feasible in patients with RAA and, therefore, remains our preferential side for the operation of all patients with EA.

\section{Disclosure Statement}

No competing financial interests exist.

\section{References}

1. Alexander F, Johanningman J, Martin LW. Staged repair improves outcome of high-risk premature infants with esophageal atresia and tracheoesophageal fistula. J Pediatr Surg 1993;28:151-154.

2. Myers NA. The history of oesophageal atresia and tracheooesophageal fistula, 1670-1984. Prog Pediatr Surg 1986;20: 106-157.

3. Harrison MR, Hanson BA, Mahour GH. The significance of right aortic arch in repair of esophageal atresia and tracheoesophageal fistula. J Pediatr Surg 1977;12:861-869.

4. Allen SR, Ignacio R, Falcone RA. The effect of a right-sided aortic arch on outcome in children with esophageal atresia and tracheoesophageal fistula. J Pediatr Surg 2006;41:479483.

5. Bowkett B, Beasley SW, Myers NA. The frequency, significance, and management of a right aortic arch in association with esophageal atresia. Pediatr Surg Int 1999;15:28-31.

6. Lillehei CW, Colan S. Echocardiography in the preoperative evaluation of vascular rings. J Pediatr Surg 1992;27:11181121.

7. Stringel G, Coln D, Guetrin L. Esophageal atresia and right aortic arch. Right or left thoracotomy? Pediatr Surg Int 1990; 5:103-105.

8. Babu R, Spitz PL, Drake DP. The management of oesophageal atresia in neonates with right-sided aortic arch. J Pediatr Surg 2000;35:56-58.

9. Holcomb GW, 3rd, Rothenberg SS, Bax KM, Martinez-Ferro M, Albanese CT, Ostlie DJ, van Der Zee DC, Yeung CK. Thoracoscopic repair of esophageal atresia and tracheoesophageal fistula: A multi-institutional analysis. Ann Surg 2005;242:422-428.

10. Shenoy VG, Jawale SA, Oak SN. Esophageal atresia with distal tracheoesophageal fistula associated with situs inversus. Pediatr Surg Int 2001;17:538-539.

Address correspondence to: Kenneth K.Y. Wong, PhD, FRCSEd, FHKAM Department of Surgery The University of Hong Kong Queen Mary Hospital Pokfulam Road Hong Kong HKG China

E-mail: kkywong@hku.hk 